\title{
Propiedades Psicométricas de la Escala de Depresión de Edimburgo en una Muestra Argentina
}

\author{
Psychometric Properties for the Edinburgh Postnatal Depression Scale in an \\ Argentinian Sample
}

\author{
Carlos Alberto López-Janer ${ }^{1}$, Pilar Quirós ${ }^{2}$ y Raúl Cabestrero ${ }^{3}$
}

\begin{abstract}
Resumen
El objetivo de este estudio fue evaluar la estructura subyacente de la EPDS mediante la comparación de cuatro modelos de análisis factorial confirmatorio, utilizando el método de máxima verosimilitud, en una muestra de 111 mujeres pertenecientes a la provincia del Chubut (Argentina). Los resultados confirmaron que el modelo con mejor ajuste a los datos fue el de tres factores: depresión, ansiedad y anhedonia $\left(\square^{2}=37,389, p=.235\right.$; RMSEA $=0,039,90 \%$ CI $=0-0,08$; CFI $=0,978$ y NNFI $\left.=0,969\right)$. La consistencia interna de la escala fue aceptable: $\alpha$ de Cronbach=.75. Ninguna de las variables sociodemográficas registradas se asoció con la depresión posparto, exceptuando la situación laboral. Las mujeres que trabajaban puntuaron significativamente más alto $(M=8,47 ; D T=4,33)$ en la escala que las que estaban en situación de desempleo $(\mathrm{M}=6,30 ; \mathrm{DT}=3,31)$. Los resultados obtenidos apoyan el carácter multidimensional de la EPDS.
\end{abstract}

Palabras clave: análisis factorial confirmatorio, anhedonia, ansiedad, depresión, EPDS

\begin{abstract}
This study aimed to evaluate the underlying structure of the EPDS by comparing four models of confirmatory factor analysis, using the maximum likelihood method, in a sample of 111 women from the Chubut province (Argentina). The results confirmed that the best fitting model was the three factors one: depression, anxiety and anhedonia $\left(\square^{2}=37.389, p=.235\right.$; RMSEA $=0.039,90 \% \mathrm{CI}=0-0.08$; IFC $=0.978$ and NNFI=0.969). The scale showed an acceptable internal consistency value: Cronbach's $\alpha=.75$. None of the registered sociodemographic variables was associated with postnatal depression, except for the work status. Working women scored significantly higher $(\mathrm{M}=8.47 ; \mathrm{SD}=4.33)$ on the scale than those unemployed $(\mathrm{M}=6.30 . \mathrm{SD}=3.31)$. The results support the idea of the multidimensional nature of the EPDS.
\end{abstract}

Keywords: anhedonia, anxiety, confirmatory factor analysis, depression, EPDS

\footnotetext{
1 Universidad del Chubut, Rawson, Chubut, Argentina. Investigador en Formación en la Escuela Internacional de Doctorado de la Universidad Nacional de Educación a Distancia (UNED), Madrid, España. Correo: clopezl1@alumno.uned.es

2 Departamento Psicología Básica II, Universidad Nacional de Educación a Distancia (UNED), Madrid, España. Correo: pquiros@psi.uned.es (autor de correspondencia)

3 Departamento Psicología Básica II, Universidad Nacional de Educación a Distancia (UNED), Madrid, España. Correo: rcabestrero@psi.uned.es

Revista Iberoamericana de Diagnóstico y Evaluación - e Avaliação Psicológica. RIDEP · Nº61 · Vol.4 · 33-47 · 2021

ISSN: 1135-3848 print /2183-6051online
} 


\section{Introducción}

La depresión posparto (DPP) es una de las complicaciones más frecuentes que se plantean tras el parto (Jardri et al., 2006), asociada con la experiencia emocional de las mujeres después de este acontecimiento. Se considera que es distinta de la denominada "tristeza materna", un periodo transitorio de disforia materna, menos definida, que ocurre unos pocos días después del parto (Bergant et al., 1999; O'Hara, 1987) y que no requiere tratamiento (Brockington, 2004). La duración de la DPP es, sin embargo, más problemática. Algunos autores han sugerido que los casos que se presentan transcurridos 6 meses después del alumbramiento no deben ser incluidos en este diagnóstico (Tuohy \& McVey, 2008). En este sentido, el Manual Diagnóstico y Estadístico de Trastornos Mentales en su quinta edición (DSM-5) incluyó como novedad frente a la versión anterior, la actualización del trastorno depresivo mayor relacionado con el parto, considerando su inicio en el periodo perinatal y su desarrollo durante el embarazo o en las primeras cuatro semanas después del parto. Si bien la consideración del inicio durante el embarazo ha sido bien aceptada, la falta de distinción en el desarrollo entre el inicio "preparto" y el inicio "posparto", ha generado cierta controversia, ya que se considera que puede haber diferencias importantes en los episodios del estado de ánimo, los cuales pueden tener etiologías distintas, así como perfiles clínicos y respuestas al tratamiento también diferentes. Además, restringir la duración a un período de 4 semanas después del parto excluiría potencialmente muchos episodios del estado de ánimo que están asociados con un inicio posparto. Por tanto, debería modificarse este criterio para poder distinguir entre episodios con inicio preparto y posparto y el marco temporal debería aumentarse de 4 semanas a 6 meses (Mendoza \& Saldivia, 2015; Sharma \& Mazmanian, 2014).

La prevalencia de la DPP se sitúa entre un 10 y un $20 \%$, según estudios realizados en países industrializados (por ej., Castañón \& Pinto (2008); Eastwood et al. (2011); Eberhard-Gran et al. (2001); Gutiérrez-Zotes et al. (2018) y Tuohy \& McVey (2008). Sin embargo, Halbreich (2005) señaló que las tasas de prevalencia reportadas podrían variar enormemente entre culturas, e incluso podrían ser diferentes dentro de una misma cultura.

El impacto de la DPP se evidencia en tres ámbitos diferentes: las madres, los niños y la familia. En el caso de los niños es clara su influencia sobre su posterior desarrollo, presentando un mayor riesgo de sufrir a largo plazo problemas conductuales, cognitivos y de interacción social (Vega-Dienstmaier et al., 2002). Dado que este tipo de depresión es habitualmente subdiagnosticada por los profesionales de la salud, resulta imprescindible disponer de buenas herramientas de diagnóstico que permitan su detección temprana con el fin de implementar el tratamiento adecuado. En este sentido, la Escala de Depresión de Edimburgo (EPDS por sus siglas en inglés), diseñada por Cox et al. (1987) es una de las más utilizadas para este cometido. Estos autores vieron la necesidad de disponer de un instrumento que permitiera evaluar los síntomas depresivos que se podían presentar tras el parto, ya que los instrumentos existentes para evaluar la depresión en la población general no resultaban válidos al incidir demasiado sobre los síntomas somáticos (por ejemplo, dificultad para dormir, pérdida de apetito o fatiga) que podían ser debidos simplemente a adaptaciones fisiológicas normales relacionadas con el propio embarazo y posterior parto.

Desde su concepción, la EPDS se ha adaptado para su uso a distintos países y se ha convertido en el instrumento más utilizado para una primera aproximación a la depresión posparto. Además de la utilización de la versión original en países de habla inglesa (Reino Unido, Estados Unidos, Canadá y Australia) ha sido traducida a más de 50 idiomas (Bergink et al., 2011) y validada en contextos socioculturales muy diferentes, como, por ejemplo, en Tailandia (Pitanupong et al., 2007), Nepal (Bhusal et al., 2016), Japón (Kubota et al., 2014), Sudáfrica (de Bruin et al., 2004), México (Oquendo et al., 2008), Chile (Alvarado et al., 2012; Castañón \& Pinto, 2008), Perú (VegaDienstmaier et al., 2002), Colombia (CampoArias et al., 2007), Brasil (Reichenheim et al., 2011), Noruega (Berle et al., 2003), Francia (Adouard et al., 2005; Guedeney \& Fermanian, 1998), Hungría (Töreki et al., 2014), Italia (Petrozzi \& Gagliardi, 2013), España (Garcia- 
Esteve et al., 2003; Gutiérrez-Zotes et al., 2018). En el trabajo de Small et al. (2007), además de utilizar la versión inglesa, incluyeron submuestras de mujeres de origen turco, filipino y vietnamita, con sus correspondientes traducciones de la escala a los distintos idiomas.

A pesar de que el uso de la EPDS está muy extendido, su estructura factorial puede variar en función de la población que se considere. Si bien la escala fue conceptualizada inicialmente como una medida unidimensional de la depresión posparto (Cox et al., 1987), tan sólo en unos pocos estudios se ha confirmado una estructura unidimensional, siendo más frecuente la solución de 2 o 3 factores (ver Tabla 1). El hecho de que la EPDS mida algo más que síntomas de depresión no resultaría extraño, dado su origen y elementos (King, 2012). Los ítems que conforman la escala se seleccionaron a partir de varias escalas combinadas de depresión y ansiedad, incluyendo la escala de irritabilidad, depresión y ansiedad (Snaith et al., 1978), la escala de ansiedad y depresión (Bedford \& Foulds, 1977) y, principalmente, la escala de ansiedad y depresión hospitalaria (HADS) (Zigmond \& Snaith, 1983). El modelo predominante de 2 factores suele incluir como factores la ansiedad y la depresión y el de 3 factores comprende, además de los dos señalados, el de anhedonia. Junto a las diferencias señaladas, hay que resaltar también las variaciones respecto a los ítems que conforman cada factor. Estas discrepancias respecto a la estructura factorial de la EPDS pueden estar reflejando importantes diferencias metodológicas (Coates et al., 2017). En muchos de los estudios se ha utilizado para la extracción de los factores el análisis de componentes principales, el cual no se puede considerar un verdadero método de análisis factorial sino una técnica distinta, cuyo objetivo principal es la reducción de datos (Ledesma et al., 2019). No distingue entre fuentes de variación comunes y únicas, y los componentes que crea incorporan ambas, incluido el error aleatorio (Costello \& Osborne, 2005; Ledesma et al., 2019; Tuohy \& McVey, 2008).

Por otra parte, las rotaciones ortogonales tampoco deberían ser utilizadas ya que sólo son adecuadas para las situaciones en las que no se espera que los factores correlacionen. No sería éste el caso del análisis factorial de la EPDS donde la ansiedad y la depresión tienen una alta comorbilidad en este tipo de población (Coates et al., 2017), siendo, por consiguiente, más apropiado aplicar las rotaciones oblicuas (Ledesma et al., 2019). Otro tema que puede afectar a las soluciones factoriales tiene que ver con el número de factores que se retienen, para lo cual se pueden utilizar distintos criterios: el de Kaiser et al. (1971) basado en autovalores $>1$ (es el que más frecuentemente se utiliza, aunque algunos autores consideran que es el menos exacto (Ledesma et al., 2019; Velicer \& Jackson, 1990), el Test de Scree (Cattell, 1966), o el análisis paralelo de Horn (1965). Esta última prueba permite obtener autovalores para cada uno de los factores y es considerada por algunos autores el criterio más objetivo (Ruiz \& San Martín, 1993). Por otra parte, el valor de la carga factorial (la correlación entre la variable y el factor) que los autores consideran apropiada determinará también si un ítem tendrá peso o no en un factor, cambiando, por tanto, la estructura factorial final. En este sentido, eligiendo cargas factoriales más altas se excluirían ítems que podrían ser incluidos en otros estudios que utilicen un punto de corte inferior. Asimismo, la estructura factorial también puede verse afectada por las características de la muestra (por ejemplo, diferencias culturales en cuanto a la expresión de los síntomas o en la propia experiencia para revelar sentimientos en una entrevista de investigación) y su tamaño (hay poco acuerdo respecto a cómo calcular los tamaños muestrales apropiados para los estudios de análisis factorial, en general se considera que, como mínimo, el tamaño de la muestra debe ser 10 veces el número de variables a analizar). Otro aspecto que puede comprometer la estructura factorial de la escala es la propia forma de cumplimentarla (autoadministrada vs. oral) o incluso el momento temporal elegido para ello. Por último, también puede estar influyendo el hecho de que la EPDS haya sido traducida del inglés a muy diferentes idiomas. El significado preciso de algunos ítems puede cambiar ligeramente, a pesar del cuidado que se pueda haber tenido al hacer la traducción (Coates et al., 2017; Small et al., 2007).

Todo ello hace difícil la comparación entre los estudios en cuanto a las soluciones factoriales halladas. Hay que tener presente, también, que 
Tabla 1. Estructuras factoriales subyacentes de la EPDS obtenidas por diversos estudios

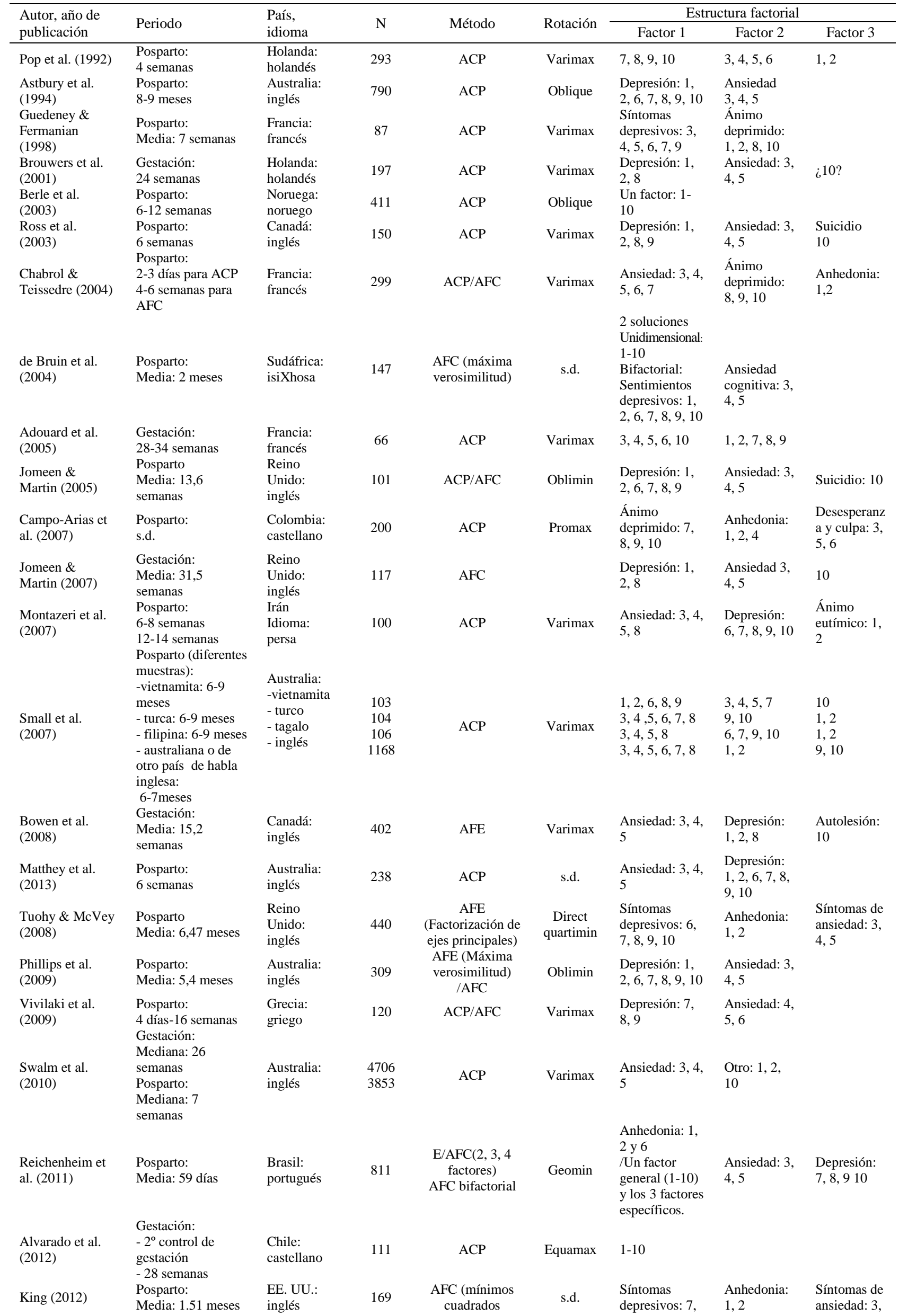




\begin{tabular}{|c|c|c|c|c|c|c|c|c|}
\hline \multirow{2}{*}{$\begin{array}{l}\text { Autor, año de } \\
\text { publicación }\end{array}$} & \multirow{2}{*}{ Periodo } & \multirow{2}{*}{$\begin{array}{l}\text { País, } \\
\text { idioma }\end{array}$} & \multirow{2}{*}{$\mathrm{N}$} & \multirow{2}{*}{ Método } & \multirow{2}{*}{ Rotación } & \multicolumn{3}{|c|}{ Estructura factorial } \\
\hline & & & & & & Factor 1 & Factor 2 & Factor 3 \\
\hline & & & & generalizados & & $8,9,10$ & & 4,5 \\
\hline $\begin{array}{l}\text { Agampodi \& } \\
\text { Agampodi } \\
(2013)\end{array}$ & $\begin{array}{l}\text { Gestación: } \\
\text { 24-36 semanas }\end{array}$ & $\begin{array}{l}\text { Sri Lanka: } \\
\text { cingalés }\end{array}$ & 376 & $\mathrm{ACP}$ & Varimax & $\begin{array}{l}\text { Anhedonia: } 1 \text {, } \\
2,8\end{array}$ & $\begin{array}{l}\text { Depresión y } \\
\text { ansiedad: } 3 \text {, } \\
4,5,6,7,8 \text {, } \\
9,10\end{array}$ & \\
\hline $\begin{array}{l}\text { Petrozzi \& } \\
\text { Gagliardi (2013) }\end{array}$ & $\begin{array}{l}\text { Posparto: } \\
2 \text { días }\left(1^{\mathrm{a}} \text { medición }\right) \\
3 \text { meses }\left(2^{\mathrm{a}}\right. \\
\text { medición })\end{array}$ & $\begin{array}{l}\text { Italia } \\
\text { italiano }\end{array}$ & 594 & $\begin{array}{c}\text { AFE } \\
\text { (Factorización de } \\
\text { ejes principales) }\end{array}$ & Promax & $\begin{array}{l}\text { Depresión: } 7 \text {, } \\
8,9,10\end{array}$ & $\begin{array}{l}\text { Ansiedad; } 3 \text {, } \\
4,5,6\end{array}$ & $\begin{array}{l}\text { Anhedonia: } \\
1,2\end{array}$ \\
\hline $\begin{array}{l}\text { Töreki et al. } \\
\text { (2013) }\end{array}$ & $\begin{array}{l}\text { Gestación: } \\
12 \text { semanas }\end{array}$ & $\begin{array}{l}\text { Hungría: } \\
\text { húngaro }\end{array}$ & 219 & $\mathrm{ACP}$ & Varimax & $2,4,5,6,10$ & $3,8,9$ & 1,7 \\
\hline $\begin{array}{l}\text { Hartley et al. } \\
(2014)\end{array}$ & $\begin{array}{l}\text { Posparto: } \\
\text { Media: } 4 \text { meses }\end{array}$ & $\begin{array}{l}\text { EE. UU.: } \\
\text { - inglés } \\
\text { - castellano }\end{array}$ & $\begin{array}{c}(220) \\
122 \\
98\end{array}$ & $\begin{array}{l}\text { AFC (máxima } \\
\text { verosimilitud) }\end{array}$ & s. d. & $\begin{array}{l}\text { Depresión: } 1 \text {, } \\
2,8,9 \\
\text { Depresión: } 1 \text {, } \\
2,8,9\end{array}$ & $\begin{array}{l}\text { Ansiedad: } 3 \text {, } \\
4,5 \\
\text { Ansiedad: } 3 \text {, } \\
4,5\end{array}$ & \\
\hline $\begin{array}{l}\text { Kubota et al. } \\
\text { (2014) }\end{array}$ & $\begin{array}{l}\text { Posparto: } \\
\text { Media: } 1 \text { mes }\end{array}$ & $\begin{array}{l}\text { Japón: } \\
\text { japonés }\end{array}$ & $\begin{array}{c}690 \\
1 / 2 \mathrm{AFE} \\
1 / 2 \mathrm{AFC}\end{array}$ & $\begin{array}{l}\text { AFE (Máxima } \\
\text { verosimilitud) } \\
\text { /AFC }\end{array}$ & Promax & $\begin{array}{l}\text { Ansiedad. 3, 4, } \\
5\end{array}$ & $\begin{array}{l}\text { Anhedonia: } \\
1,2\end{array}$ & $\begin{array}{l}\text { Depresión: } \\
7,8,9\end{array}$ \\
\hline $\begin{array}{l}\text { Töreki et al. } \\
\text { (2014) }\end{array}$ & $\begin{array}{l}\text { Posparto: } \\
6 \text { semanas }\end{array}$ & $\begin{array}{l}\text { Hungría: } \\
\text { húngaro }\end{array}$ & 266 & $\mathrm{ACP} / \mathrm{AFC}$ & Oblique & $\begin{array}{l}\text { Ansiedad: } 3,4, \\
5,6\end{array}$ & $\begin{array}{l}\text { Depresión: } \\
1,2,9,10\end{array}$ & \\
\hline $\begin{array}{l}\text { Zhong et al. } \\
\text { (2014) }\end{array}$ & $\begin{array}{l}\text { Gestación: } \\
\text { Media: 9,8 semanas }\end{array}$ & $\begin{array}{l}\text { Perú: } \\
\text { castellano }\end{array}$ & 1517 & $\begin{array}{c}\text { ACP/AFC } \\
\text { (máxima } \\
\text { verosimilitud) }\end{array}$ & Varimax & $\begin{array}{l}\text { Ansiedad y } \\
\text { depresión: } 3 \text {, } \\
4,5,6,7,8,9 \text {, } \\
10\end{array}$ & $\begin{array}{l}\text { Anhedonia: } \\
1,2\end{array}$ & \\
\hline $\begin{array}{l}\text { Bina \& } \\
\text { Harrington } \\
(2016)\end{array}$ & $\begin{array}{l}\text { Posparto } \\
6 \text { semanas }\end{array}$ & $\begin{array}{l}\text { Israel: } \\
\text { hebreo }\end{array}$ & 715 & $\mathrm{AFC}$ & s.d. & $\begin{array}{l}\text { Depresión: } 1 \text {, } \\
2,7,8,910\end{array}$ & $\begin{array}{l}\text { Ansiedad: } 3 \text {, } \\
4,5\end{array}$ & \\
\hline $\begin{array}{l}\text { Cunningham et } \\
\text { al. (2015) }\end{array}$ & $\begin{array}{l}\text { Posparto: } \\
0-13 \text { meses }\end{array}$ & $\begin{array}{l}\text { Australia: } \\
\text { inglés }\end{array}$ & 636 & $\begin{array}{l}\text { AFE (máxima } \\
\text { verosimilitud) } \\
\text { /AFC }\end{array}$ & Geomin & $\begin{array}{l}\text { Admisión: } \\
1,2,3,6,7,8, \\
9,10 \\
\text { Alta: } \\
1,2\end{array}$ & $\begin{array}{l}\text { Admisión: } \\
3,4,5 \\
\text { Alta: } \\
3,4,5,6\end{array}$ & $\begin{array}{l}\text { Alta: } \\
7,8,9,10\end{array}$ \\
\hline $\begin{array}{l}\text { Kwan et al. } \\
(2014)\end{array}$ & $\begin{array}{l}\text { Gestación: } \\
\text { 26-28 semanas }\end{array}$ & $\begin{array}{l}\text { Singapur: } \\
\text { - inglés } \\
\text { - tamil } \\
\text { - malayo } \\
\text { - chino }\end{array}$ & 920 & $\begin{array}{l}\text { AFE/ (mínimos } \\
\text { cuadrados } \\
\text { ponderados) } \\
\text { /AFC }\end{array}$ & Geomin & $1-10$ & & \\
\hline $\begin{array}{l}\text { Odalovic et al. } \\
(2015)\end{array}$ & $\begin{array}{l}\text { Gestación: } \\
\text { Media: } 25,7 \\
\text { semanas } \\
\text { Posparto: } \leq 28 \\
\text { semanas }\end{array}$ & $\begin{array}{l}\text { Serbia: } \\
\text { serbio }\end{array}$ & $\begin{array}{c}(201) \\
76 \\
125\end{array}$ & $\mathrm{ACP} / \mathrm{AFC}$ & Varimax & $\begin{array}{l}3,4,5 \\
3,4,5\end{array}$ & $\begin{array}{l}7,9,10 \\
7,9,10\end{array}$ & $\begin{array}{l}1,2 \\
1,2\end{array}$ \\
\hline $\begin{array}{l}\text { Coates et al. } \\
(2017)\end{array}$ & $\begin{array}{l}\text { Gestación: } \\
18 \text { y } 32 \text { semanas } \\
\text { Posparto: } \\
8 \text { semanas } \\
8 \text { meses }\end{array}$ & $\begin{array}{l}\text { Reino } \\
\text { Unido: } \\
\text { inglés }\end{array}$ & $\begin{array}{l}>11000 \\
1 / 2 \mathrm{AFE} \\
1 / 2 \mathrm{AFC}\end{array}$ & $\begin{array}{l}\text { AFE (Máxima } \\
\text { verosimilitud) } \\
\text { /AFC }\end{array}$ & Oblimin & $\begin{array}{l}\text { Depresión: } 7 \text {, } \\
8,9,10\end{array}$ & $\begin{array}{l}\text { Anhedonia: } \\
1,2\end{array}$ & $\begin{array}{l}\text { Ansiedad: } 3 \text {, } \\
4,5,6\end{array}$ \\
\hline $\begin{array}{l}\text { Gutiérrez-Zotes } \\
\text { et al. (2018) }\end{array}$ & $\begin{array}{l}\text { Posparto: } \\
\text { Media: } 32 \text { semanas }\end{array}$ & $\begin{array}{l}\text { España: } \\
\text { castellano }\end{array}$ & 1204 & $\begin{array}{l}\text { AFE/AFC } \\
\text { (mínimos } \\
\text { cuadrados no } \\
\text { ponderados) }\end{array}$ & Oblimin & $\begin{array}{l}\text { Depresión: } 7 \text {, } \\
8,9\end{array}$ & $\begin{array}{l}\text { Ansiedad: } 3 \text {, } \\
4,5,6\end{array}$ & $\begin{array}{l}\text { Anhedonia: } \\
1,2,10\end{array}$ \\
\hline $\begin{array}{l}\text { Kubota et al. } \\
(2018)\end{array}$ & $\begin{array}{l}\text { Gestación (inicio y } \\
\text { final): } \\
\text { - Media: } 25,1 \\
\text { semanas } \\
\text { - Media: } 36,2 \\
\text { semanas } \\
\text { Posparto: } \\
\text { - Media: } 32,5 \text { días }\end{array}$ & $\begin{array}{l}\text { Japón: } \\
\text { japonés }\end{array}$ & $\begin{array}{l}1075 \\
30 \% \\
\mathrm{AFE} \\
70 \% \\
\mathrm{AFC}\end{array}$ & $\begin{array}{c}\text { AFE/AFC } \\
\text { (multigrupo) }\end{array}$ & s.d. & Ansiedad: 4,5 & $\begin{array}{l}\text { Depresión: } \\
7,9\end{array}$ & $\begin{array}{l}\text { Anhedonia: } \\
1,2\end{array}$ \\
\hline
\end{tabular}

algunos de ellos se han limitado a efectuar un análisis exploratorio en lugar de llevar a cabo, además, un análisis factorial confirmatorio basado en los resultados empíricos del primero que permitiera examinar la validez estructural de la escala. Este último permitiría (basándose en los índices de ajuste habituales) comprobar el ajuste del modelo a los datos obtenidos. En la Tabla 1 se puede apreciar la diversidad de soluciones factoriales obtenidas en distintos estudios.
Por otra parte, conviene tener en cuenta que no existen muchos estudios que hayan evaluado la estructura subyacente de la EPDS en poblaciones con características étnicas y socioeconómicas diversas. Una mejor comprensión de la estructura factorial de la EPDS mediante el uso del análisis factorial confirmatorio con este tipo de muestras poco estudiadas resulta fundamental para el uso futuro de la escala en la investigación. Si la estructura factorial varía entre las muestras, las 
Tabla 2. Datos sociodemográficos de la muestra.

\begin{tabular}{lcc}
\hline Variables & Frecuencia & Porcentaje \\
\hline Edad de la madre & & \\
M: 25,22 años & & \\
DT: 6,38 & 21 & $21,2 \%$ \\
$\quad<20$ años & 59 & $52,3 \%$ \\
$21-30$ años & 25 & $22,5 \%$ \\
$\quad>30$ años & & \\
Edad del bebé & & \\
M: 1,21 meses & & $65,77 \%$ \\
DT: 1,32 & 73 & $26,13 \%$ \\
$\quad$ < mes & 29 & $8,1 \%$ \\
$1-3$ meses & 9 & \\
$3-6$ meses & & $28,8 \%$ \\
Nivel de estudios & 32 & $61,3 \%$ \\
Primarios & 68 & $9,9 \%$ \\
Secundarios & 11 & $15,3 \%$ \\
Universitarios & & $84,7 \%$ \\
Situación Laboral & 17 & \\
Trabajando & 94 & $40,5 \%$ \\
Parado & & $41,44 \%$ \\
Número de hijos anteriores & 45 & $8,11 \%$ \\
0 & 46 & $46,8 \%$ \\
1-2 & 9 & $43,2 \%$ \\
$\quad>2$ & & \\
Embarazo deseado & 52 & $55,0 \%$ \\
Sí & 48 & $45,0 \%$ \\
No & 61 &
\end{tabular}

puntuaciones resultarán poco discriminativas para medir los distintos niveles de síntomas y, en consecuencia, no permitirán comparar los resultados obtenidos entre los diferentes estudios (King, 2012).

En este sentido, el objetivo del estudio que se propone es validar la EPDS y analizar su estructura factorial mediante el análisis factorial confirmatorio (a través de los modelos más frecuentemente encontrados en la literatura y reflejados en la Tabla 1) en Argentina, donde, debido a su extenso territorio, habitado por comunidades separadas en algunos casos por miles de kilómetros, coexisten poblaciones con notables diferencias culturales y lingüísticas, entre otras. El trabajo se plantea como un estudio inicial, circunscrito a una región del sur de Argentina, en concreto, limitándose a las ciudades de Rawson y Trelew (pertenecientes a la provincia del Chubut).

\section{Método}

\section{Participantes}

Se tomó una muestra de conveniencia de 111 mujeres de entre las que acudían a dos Centros de Salud Pública: Hospital Santa Teresita de Rawson y Centro Materno Infantil del Hospital de Trelew para hacer los seguimientos ordinarios de control postparto de las madres y sus hijos. Todas ellas participaron voluntariamente en el estudio, una vez que éste recibió la aprobación de los Comités de Ética de la Investigación de ambos Centros de Salud y fuera autorizado por sus directores (el procedimiento se ajustó a la Declaración de Helsinki, 1964) y que las participantes hubieran firmado el consentimiento informado. El tamaño de la muestra se estimó teniendo en cuenta que debe ser al menos 10 veces el número de variables a analizar (Alvarado et al., 2012; Small et al., 2007).

Como criterios de inclusión se establecieron los siguientes: que supieran leer y escribir correctamente en castellano, que el parto hubiera sido a término y no gemelar, que no hubieran tenido un aborto en el último año, que no tuvieran antecedentes de enfermedades psiquiátricas y/o neurológicas graves ni historia de consumo de sustancias psicoactivas. Las mujeres no recibieron ningún tipo de incentivo por su participación.

Los datos sociodemográficos de las participantes pueden verse en la Tabla 2 . 


\section{Instrumentos}

Escala de Depresión de Edimburgo. Se trata de una escala autoadministrada que consta de 10 ítems, que son puntuados en una escala tipo Likert de 4 puntos (de 0 a 3), siendo el rango de puntuación total de 0 a 30. Por lo general, una puntuación $\geq 10$ se considera aceptable para discriminar a personas con posible sintomatología. Una puntuación $\geq 13$ ya debería ser considerada desde un punto de vista clínico (Berle et al., 2003). La escala ha mostrado buenas propiedades psicométricas en la población a la que va dirigida (el $\alpha$ de Cronbach en el estudio original de Cox et al. (1987), fue de .87). Resulta fácil de cumplimentar (tan solo se precisa entre 5 y 10 minutos), no requiere experiencia psiquiátrica especializada por parte de los trabajadores sanitarios que pueden encargarse de administrarla y es bien aceptada por las mujeres.

\section{Procedimiento}

A las mujeres participantes se les dio una breve explicación de los objetivos de la investigación $\mathrm{y}$, tras la firma del consentimiento informado, procedieron a cumplimentar de forma individual un cuestionario elaborado ad hoc para este estudio que incluía información sobre las variables sociodemográficas (por ejemplo, la edad, el estado civil, nivel educativo, la situación laboral, número de embarazos previos, etc.), las cuales pueden observarse en la Tabla 2). Seguidamente se les facilitó en mano la versión en castellano de la EPDS (Garcia-Esteve et al., 2003) siendo la aplicación de la escala de forma autoadministrada (aunque uno de los investigadores presentes podía clarificar las posibles dudas que pudieran surgir). En ella se pregunta a las mujeres sobre cómo se han sentido en los últimos 7 días. El tiempo medio de la recogida de todos los datos fue de 10 minutos, procediéndose ésta, para cada participante, en distintos momentos después del parto $(\mathrm{M}=1.21$ meses; DT=1.32). La puntuación total de la escala de la muestra seleccionada estuvo comprendida en un rango de 0 a $16(\mathrm{M}=6.63$; $\mathrm{DT}=3.55)$.

Una vez recogidos todos los datos, se agradeció a las mujeres su participación y se les indicó que se les facilitaría, en el caso de que estuvieran interesadas, información sobre los resultados de la investigación.

\section{Análisis de datos}

Los análisis estadísticos fueron realizados con el paquete estadístico SPSS v25, considerando significativo un valor de $p=.05$.

Estructura factorial de la EPDS. Por lo que respecta a la estructura factorial de la escala, se llevó a cabo un análisis factorial confirmatorio empleando el método de máxima verosimilitud mediante el programa AMOS 25.0, explorando diferentes soluciones (de 1 a 3 factores: Modelos 1, 2a, 2b y 3 -ver Tabla 3-), en base a los diferentes modelos encontrados en la literatura y recogidos en la Tabla 1.

Debido a que la prueba chi-cuadrado $\left(\square^{2}\right)$ es muy sensible al tamaño de la muestra, la evaluación de los modelos se realizó mediante una combinación de índices de ajuste, utilizándose el error cuadrático medio de aproximación (RMSEA), como índice de valor absoluto, el índice de ajuste comparativo (CFI) y el índice no normalizado de ajuste (NNFI) o índice de TuckerLewis (TLI), como índices de ajuste incremental. Los índices de valor absoluto evalúan el grado en el que la matriz de covarianza implícita del modelo coincide con la matriz de covarianza observada. Se mide la falta de ajuste, por tanto, cuanto mejor se ajusta el modelo a los datos, menores valores tendrán estos índices, indicando un valor de 0 un ajuste óptimo. Por el contrario, los índices de ajuste incrementales evalúan el grado en el que el modelo que se somete a prueba es superior a un modelo alternativo en la reproducción de la matriz de covarianza observada. Evalúan la bondad de ajuste, por consiguiente, cuanto mayor sea el valor de estos índices, mejor será el ajuste del modelo, considerando un valor de 1 un ajuste óptimo (Chen, 2007). Los valores críticos de estos índices (al no ser estadísticos) que han sido consensuados por la comunidad científica son los siguientes: en el caso del RMSA, valores inferiores a .05 indican un ajuste perfecto, de .05 a .08 un ajuste adecuado, de .08 a .10 un ajuste mediocre y valores superiores a .10 indicarían un ajuste deficiente, por lo que el modelo debería ser rechazado. Por lo que respecta al CFI y al NNFI, los valores comprendidos entre .90 y .95 indican un ajuste del modelo aceptable. Valores superiores a .95 son considerados como un ajuste adecuado (Hu \& Bentler, 1999). 
Tabla 3. Resultados del análisis factorial confirmatorio para cada uno de los modelos

\begin{tabular}{|c|c|c|c|c|c|c|c|c|c|c|c|c|}
\hline Modelo & Factor 1 & Factor 2 & Factor 3 & $c^{2}$ & $\mathrm{gl}$ & $p$ & RMSEA & $90 \%$ IC & $\triangle \mathrm{RMSEA}$ & CFI & $\Delta \mathrm{CFI}$ & NNFI \\
\hline $\begin{array}{l}\text { Modelo 1: } \\
\text {-Cox et al. (1987) } \\
\text {-Berle et al. (2003) } \\
\text {-Reichenheim et al. } \\
\text { (2011) } \\
\text {-Alvarado et al. } \\
\text { (2012) } \\
\text {-Kwan et al. (2014) }\end{array}$ & $\begin{array}{l}\text { Depresión } \\
1-10\end{array}$ & & & 55.034 & 35 & .017 & .072 & $.03-.11$ & .033 & .918 & .06 & .894 \\
\hline $\begin{array}{l}\text { Modelo 2a: } \\
\text {-Zhong et al. (2014) }\end{array}$ & $\begin{array}{c}\text { Ansiedad } \\
\text { Depresión 3-10 }\end{array}$ & $\begin{array}{l}\text { Anhedonia } \\
1 \text { y } 2\end{array}$ & & 52.371 & 34 & .023 & .072 & $.03-.11$ & .031 & .925 & .053 & .900 \\
\hline $\begin{array}{l}\text { Modelo 2b: } \\
\text {-Astbury et al. } \\
\text { (1994) } \\
\text {-de Bruin et al. } \\
\text { (2004) } \\
\text {-Phillips et al. } \\
\text { (2009) } \\
\text {-Bina \& Harrington } \\
(2016)\end{array}$ & $\begin{array}{c}\text { Depresión } 1,2 \text {, } \\
6,7,8,9,10\end{array}$ & $\begin{array}{c}\text { Ansiedad } \\
3,4,5\end{array}$ & & 45.363 & 34 & .092 & .055 & $0-.09$ & .016 & .953 & .025 & .938 \\
\hline $\begin{array}{l}\text { Modelo 3.: } \\
\text {-Pop et al. (1992) } \\
\text {-Petrozzi and } \\
\text { Gagliardi (2013) } \\
\text {-Cunningham et al. } \\
\text { (2015) } \\
\text {-Coates et al. (2017) }\end{array}$ & $\begin{array}{l}\text { Depresión } \\
7,8,9,10\end{array}$ & $\begin{array}{l}\text { Ansiedad } \\
3,4,5,6\end{array}$ & $\begin{array}{c}\text { Anhedonia } \\
1,2\end{array}$ & 37.389 & 32 & .235 & .039 & $0-.08$ & & .978 & & .969 \\
\hline
\end{tabular}

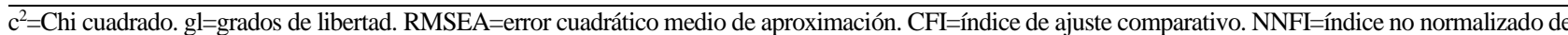
ajuste. $\triangle$ RMSEA=incremento de RMSEA respecto al último modelo. $\triangle \mathrm{CFI}=$ incremento de $\mathrm{CFI}$ respecto al último modelo. IC=intervalo de confianza

\section{Resultados}

Los resultados del análisis factorial confirmatorio para cada uno de los modelos se presentan en la Tabla 3.

Analizando los modelos individualmente, se observa que el Modelo 1 mostraría un ajuste adecuado a los datos respecto a RMSEA, aceptable en relación con el CFI e inadecuado teniendo en cuenta el NNFI, ya que el valor alcanzado (.894 está por debajo del punto de corte inferior considerado aceptable de .90). El Modelo 2a (factores: ansiedad-depresión y anhedonia) se considera adecuado a los datos respecto a los índices de ajuste considerados. Un mejor ajuste se observa en el Modelo 2b (factores: depresión y ansiedad). Sin embargo, el modelo que presenta el mejor ajuste es el de tres factores (depresión, ansiedad y anhedonia). Este modelo se puede observar en la Figura 1.

Para comparar el modelo con mejor ajuste a los datos (Modelo 3) con el resto de los modelos, se tuvieron en cuenta los valores del $\triangle$ RMSEA y del $\triangle \mathrm{CFI}$, en lugar del utilizado tradicionalmente de $\Delta \chi^{2}$ (por las razones comentadas anteriormente de la sensibilidad del estadístico $\chi^{2}$ al tamaño de la muestra). Considerando que incrementos en estos índices $\leq .01$ indicarían que no hay mejora en el

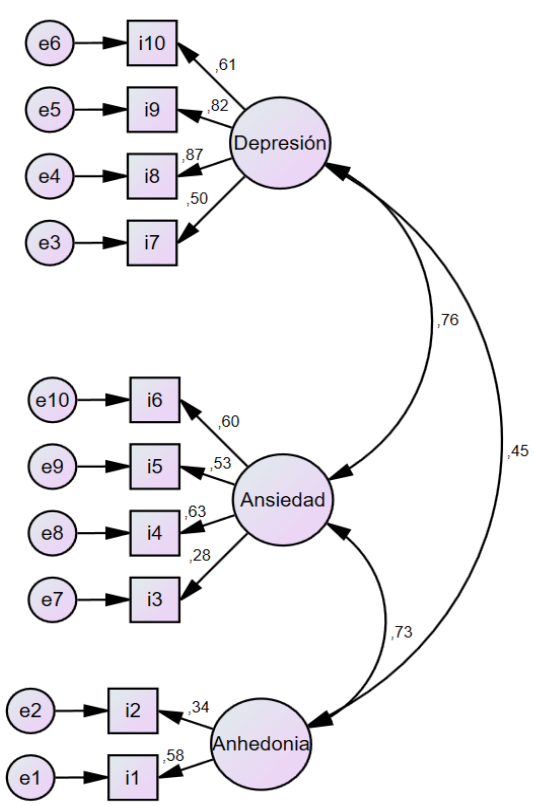

Figura 1. Modelo de tres factores de la EPDS con pesos factoriales estandarizados en cada factor

ajuste, por lo que los modelos podrían considerarse iguales (Cheung \& Rensvold, 2002), se observa que los incrementos son todos >.01, por lo que el modelo que mejor se ajusta a los datos observados es el de 3 factores.

Fiabilidad de los ítems de la EPDS. En la Tabla 4 se muestra la fiabilidad total de la escala 
Tabla 4. Valores del $\alpha$ de Cronbach para los ítems de la EPDS.

\begin{tabular}{lcc}
\hline Ítems de la EPDS & \multicolumn{2}{c}{ Valor del $\alpha$ de Cronbach } \\
\cline { 2 - 3 } i1. He sido capaz de reír y ver el lado bueno de las cosas & .73 & Subescalas \\
i2. He mirado el futuro con placer & .75 & Anhedonia \\
i3. Me he culpado sin necesidad cuando las cosas no salían bien & .76 & .32 \\
i4. He estado ansiosa y preocupada sin motivo & .71 & .72 \\
i5. He sentido miedo y pánico sin motivo alguno & .71 \\
i6. Las cosas me oprimen o agobian & .73 \\
i7. Me he sentido tan infeliz que he tenido dificultad para dormir & .70 \\
i8. Me he sentido triste y desgraciada & .69 \\
i9. He sido tan infeliz que he estado llorando & .74 \\
i10. He pensado en hacerme daño a mí misma & .75 \\
Total EPDS & Ansiedad \\
\hline
\end{tabular}

$(\alpha=.75)$, la fiabilidad de ésta al eliminar un determinado ítem, así como la fiabilidad de cada una de las tres subescalas. Como se puede observar, la fiabilidad total de la escala aumenta ligeramente sólo si se elimina el ítem 3. La consistencia interna de la EPDS fue evaluada también mediante el método de las dos mitades de Guttman, obteniéndose un valor de .66.

\section{Análisis de la puntuación total de la EPDS.}

La media de la puntuación total de la escala fue de 6.63 (DT=3.55). Considerando, según la mayoría de los estudios revisados, una puntuación $\geq 10$ como indicativa de presencia de síntomas depresivos, un $15.3 \%$ de las mujeres reunían este requisito y un $9.9 \%$ alcanzaron una puntuación $>$ de 13, puntuación que sugiere un riesgo alto de sufrir una depresión.

En cuanto al análisis de los datos demográficos, se realizaron una serie de ANOVAs univariados con el objeto de comprobar la existencia de diferencias significativas entre los diferentes grupos formados en cada una de las variables registradas (ver Tabla 2). Los resultados no mostraron diferencias significativas entre los distintos niveles de las variables, salvo en el caso de la situación laboral $\left(F_{1,109}=5.621, p=.02\right.$, $\eta_{\mathrm{p}}^{2}=.49$ ). Las mujeres que trabajan puntuaron más alto $(M=8.47$; $D T=4.33)$ en la escala que aquellas que estaban en situación de desempleo $(M=6,30$; $\mathrm{DT}=3.31$ ).

\section{Discusión}

El objetivo de este estudio fue evaluar la estructura subyacente de la EPDS, en una región concreta de Argentina, mediante la comparación de cuatro modelos de análisis factorial confirmatorio (comprendiendo de 1 a 3 factores).
Los resultados obtenidos confirmaron el carácter multidimensional de la escala, siendo el modelo de tres factores: ansiedad, (ítems 3, 4, 5, 6), anhedonia (ítems 1 y 2) y depresión (ítems 7 , 8,9 y 10), el que mejor se ajustaba a los datos. Esta estructura factorial coincide con la obtenida por Pop et al. (1992); Petrozzi and Gagliardi (2013); Cunningham et al. (2015) y Coates et al. (2017). Es la misma, también (exceptuando la inclusión del ítem 10 en el factor de anhedonia) que la obtenida en el estudio de validación de la EPDS en la población española (Gutiérrez-Zotes, 2018).

Respecto a los ítems que conforman cada factor $\mathrm{y}$, teniendo en cuenta las soluciones factoriales a las que se ha llegado en los distintos modelos presentados en la Tabla 1, se puede observar que algunos ítems se sitúan generalmente en un mismo factor. Así, los ítems 3, 4, 5 suelen estar presentes en el factor ansiedad y los ítems 7 , 8 y 9 en el de depresión. El resto de ellos van fluctuando entre los distintos factores, pudiendo ser más sensibles a influencias culturales. En este sentido, el ítem 6 se puede encontrar incluido tanto en el factor depresión como en el de ansiedad. Posiblemente, la redacción de este ítem (Las cosas me oprimen o agobian) esté abierto a distintas intepretaciones que sugieran o bien depresión o bien ansiedad (Coates et al., 2017). Esta puede haber sido también la causa de que, en este estudio, el ítem 3 (Me he culpado sin necesidad cuando las cosas no salían bien) sea el que tiene la correlación más baja con su factor (.28) y que la fiabilidad total de la escala se incremente ligeramente cuando se elimina. Los ítems que presentan una correlación más alta con cada uno de los factores son el 8 y el 9 en relación con la depresión (.87 y .82 respectivamente), el 4 y el 6 con la ansiedad (.63 y .60) y el 1 con la 
anhedonia (.58). El ítem 2 presenta, sin embargo, una correlación baja $(0,34)$, posiblemente porque, en la redacción del ítem (He mirado el futuro con placer), el término placer sea difícil de interpretar. Respecto a este factor, parecen existir dudas sobre si realmente constituiría un factor o ser consecuencia de la construcción de la propia escala, ya que los ítems que lo conforman (1 y 2) son los únicos que están redactados en forma positiva. Se considera que los elementos de redacción positiva formarían un factor separado de los elementos de redacción negativa. Por tanto, se necesitaría aportar mayor investigación para determinar si el factor anhedonia es significativo o su presencia se debe a la forma en la que está construida la escala (Mook et al., 1991). Asimismo, suele ser deseable que los factores tengan al menos tres elementos (Costello \& Osborne, 2005). Por lo que respecta al ítem 10 (He pensado en hacerme daño a mí misma) presenta una correlación alta $(0,61)$ con el factor de depresión (en otros estudios este ítem es el que tiene un menor peso en este factor - por ej., en el de Coates et al., 2017-). Hay que tener en cuenta que el $9.9 \%$ de la muestra obtuvo una puntuación $>$ de 13 en la escala y esta puntuación debería ser ya considerada desde un punto de vista clínico (Berle et al., 2003). En algunos estudios (Bowen et al., 2008; Jomen \& Martin 2005, 2007; Ross, 2003; Small et al., 2007) el ítem 10 constituye un factor independiente. Las puntuaciones en este ítem podrían ser indicativas de que las mujeres necesitan una derivación inmediata a un servicio especializado (Coates et al. 2017).

En cuanto a la fiabilidad de la escala, ésta resultó ser adecuada para la muestra seleccionada ( $\alpha$ de Cronbach=.75 -valor próximo al encontrado en otros estudios, como los de Töreki et al., 2014 y Campo-Arias et al., 2007-). Considerando la fiabilidad de cada una de las subescalas, sólo la de depresión obtuvo un valor $>.70$, mientras que los valores de las otras dos subescalas están por debajo de los que se consideraría aceptable. A este respecto, es necesario tomar en consideración, también, lo indicado anteriormente respecto al factor anhedonia y al hecho de que debería tener, al menos, tres ítems. En consecuencia, estas subescalas no podrían ser utilizadas de forma independiente como escalas de evaluación. En cualquier caso, estos resultados podrían estar condicionados por el tamaño reducido de la muestra.

Una de las limitaciones de este estudio es, precisamente, el tamaño de la muestra que no ha permitido hacer, por ejemplo, análisis multigrupo. Este será el objetivo de un trabajo posterior en el que, además, se valore la presencia de posibles síntomas depresivos no sólo en el posparto, sino también durante la gestación (Coates et al., 2017; Kubota et al., 2018), considerando estos dos periodos como especialmente vulnerables. Los cambios hormonales que se producen durante estas etapas pueden variar la susceptibilidad de las mujeres a desarrollar una depresión. Por este motivo, sería conveniente que se cumplimentara la EPDS en cada uno de los trimestres del embarazo (Al-Hashimi et al., 2019; Vazquez \& Míguez, 2019), así como en diferentes momentos después del mismo. La detección de la depresión durante el embarazo es de vital importancia ya que afecta, tanto al propio curso de éste, como al desarrollo del feto, siendo, además un potente predictor de la depresión en el posparto (Vazquez \& Míguez, 2019). Otra limitación de este estudio es el no haber contado con un patrón de oro que permitiera establecer los puntos de corte adecuados en la EPDS para la valoración de la depresión en la muestra concreta con la que se pretende trabajar (por ejemplo, la entrevista clínica estructurada para el DSM-5 - SCID—, la cual ha sido utilizada con frecuencia en los estudios de validación de la EPDS), mediante el cálculo de las áreas bajo las curvas ROC, tratando de buscar el mejor equilibrio entre la especificidad y sensibilidad de la escala en diferentes puntos de corte y tomando en consideración el valor predictivo negativo.

Por otra parte, antes de emprender este futuro trabajo, es preciso tener en cuenta las particularidades que puede haber entre el castellano hablado en España y en Argentina (que puede dar lugar a diversas interpretaciones en cuanto a la forma en la que están redactados los ítems). En este sentido, resulta necesaria la adaptación transcultural de la EPDS a la población diana, siguiendo el procedimiento de adaptación de una cultura a otra de Balluerka et al. (2007). El objetivo final sería conseguir que la EPDS pueda formar parte de un protocolo de 
cribado para la detección precoz de la depresión, tanto pre como postnatal, sobre todo en colectivos especialmente vulnerables como madres adolescentes o con recursos económicos limitados, con objeto de que se puedan establecer las medidas oportunas antes de que llegue a instaurarse.

En conclusión, aunque se haya aportado nueva evidencia del carácter multidimensional de la EPDS y los datos obtenidos se ajusten a un modelo de tres factores (depresión, ansiedad y anhedonia), los coeficientes de fiabilidad de los factores no permiten que la escala pudiera ser utilizada para evaluar los diferentes componentes de la depresión postparto. Se necesitan emprender nuevas investigaciones que tengan en cuenta las limitaciones señaladas: tamaño de la muestra (los modelos con los que se ha comparado han utilizado un $\mathrm{N}$ mucho mayor -más de 11000 en el caso del trabajo de Coates et al., 2017-), adaptación transcultural de la escala y contar con un patrón de oro que permita establecer los puntos de corte para la población con la que se pretende trabajar.

\section{Referencias}

Adouard, F., Glangeaud-Freudenthal, N. M., \& Golse, B. (2005). Validation of the Edinburgh postnatal depression scale (EPDS) in a sample of women with high-risk pregnancies in France. Archives of Women's Mental Health, 8(2), 89-95.

https://doi.org/http://doi.org/10.1007/s00737005-0077-9

Agampodi, S. B., \& Agampodi, T. C. (2013). Antenatal depression in Anuradhapura, Sri Lanka and the factor structure of the Sinhalese version of Edinburgh post partum depression scale among pregnant women. PLoS ONE, 8(7), e69708. https://doi.org/10.1371/journal.pone.0069708

Al-Hashimi, F. J. G., Kareem Alalaf, S., \& Al Tawil, N. G. (2019). Screening for depression during pregnancy using the Kurdish version of the Edinburgh Postnatal Depression Scale in Erbil city. Health Care Women International, $1-15$.

https://doi.org/10.1080/07399332.2019.1571592
Alvarado, R., Guajardo, V., Rojas, G., \& Jadresic, E. (2012). Validación de la escala de Edimburgo para embarazadas: Chile. Universidad de Chile. Facultad de Medicina.

Astbury, J., Brown, S., Lumley, J., \& Small, R. (1994). Birth events, birth experiences and social differences in postnatal depression. Australian Jorunal of Public Health, 18(2), 176-184.

https://doi.org/10.1111/j.17536405.1994.tb00222.x

Balluerka, N., Gorostiaga, A., Alonso-Arbiol, I., \& Haranburu, M. (2007). La adaptación de instrumentos de medida de unas culturas a otras: Una perspectiva práctica $=$ Test adaptation to other cultures: A practical approach. Psicothema, 19(1), 124-133.

Bedford, A., \& Foulds, G. A. (1977). Validation of the delusions- symptoms- states inventory. British Journal of Medical Psychology, 50(2), 163-171.

Bergant, A. M., Heim, K., Ulmer, H., \& Illmensee, K. (1999). Early postnatal depressive mood: Associations with obstetric and psychosocial factors. Journal of Psychosomatic Research, 46(4), 391-394. https://doi.org/10.1016/s0022-3999(98)00116-0

Bergink, V., Kooistra, L., Lambregtse-van den Berg, M. P., Wijnen, H., Bunevicius, R., van Baar, A., \& Pop, V. (2011). Validation of the Edinburgh Depression Scale during pregnancy. Journal of Psychosomatic Research, 70(4), 385-389.

https://doi.org/10.1016/j.jpsychores.2010.07.008

Berle, J. Ø., Aarre, T. F., Mykletun, A., Dahl, A. A., \& Holsten, F. (2003). Screening for postnatal depression: Validation of the Norwegian version of the Edinburgh Postnatal Depression Scale, and assessment of risk factors for postnatal depression. Journal of Affective Disorders, 76(1-3), 151-156. https://doi.org/10.1016/s0165-0327(02)00082-4

Bhusal, B. R., Bhandari, N., Chapagai, M., \& Gavidia, T. (2016). Validating the Edinburgh Postnatal Depression Scale as a screening tool for postpartum depression in Kathmandu, Nepal. International Journal of Mental Health Systems, 10, 71. https://doi.org/https://doi.org/10.1186/s13033016-0102-6 
Bina, R., \& Harrington, D. (2016). The Edinburgh Postnatal Depression Scale: Screening tool for postpartum anxiety as well? Findings from a Confirmatory Factor Analysis of the Hebrew Version. Maternal and Child Health Journal 20(4), 904-914. https://doi.org/10.1007/s10995-015-1879-7

Bowen, A., Bowen, R., Maslany, G., \& Muhajarine, N. (2008). Anxiety in a socially high-risk sample of pregnant women in Canada. Canadian Journal of Psychiatry, 53(7), 435-440. https://doi.org/10.1177/070674370805300708

Brockington, I. (2004). Postpartum psychiatric disorders. Lancet, 363(9405), 303-310. https://doi.org/10.1016/s0140-6736(03)15390-1

Brouwers, E. P. M., van Baar, A. L., \& Pop, V. J. M. (2001). Does the Edinburgh Postnatal Depression Scale measure anxiety? Journal of Psychosomatic Research, 51(5), 659-663. https://doi.org/10.1016/s0022-3999(01)00245-8

Campo-Arias, A., Ayola-Castillo, C., PeinadoValencia, H. M., Amor-Parra, M., \& Cogollo, Z. (2007). Escala de Edinburgh para depresión posparto: Consistencia interna y estructura factorial en mujeres embarazadas de Cartagena, Colombia. Revista Colombiana de Obstetricia y Ginecología, 58(4), 277-283.

Castañón, C., \& Pinto, J. (2008). Mejorando la pesquisa de depresión posparto a través de un instrumento de tamizaje, la escala de depresión posparto de Edimburgo. Revista médica de Chile, 136(7), 851-858. https://scielo.conicyt.cl/pdf/rmc/v136n7/art05. pdf

Cattell, R. B. (1966). The Scree Test for the number of factors. Multivariate Behavioral Research, 1(2), 245-276. https://doi.org/10.1207/s15327906mbr0102_10

Chabrol, H., \& Teissedre, F. (2004). Relation between Edinburgh Postnatal Depression Scale scores at 2-3 days and 4-6 weeks postpartum. Journal of Reproductive and Infant Psychology, 22(1), 33-39. https://doi.org/10.1080/02646830310001643067

Chen, F. F. (2007). Sensitivity of goodness of fit indexes to lack of measurement invariance. Structural Equation Modeling-a Multidisciplinary Journal, 14(3), 464-504. https://doi.org/10.1080/10705510701301834
Cheung, G. W., \& Rensvold, R. B. (2002). Evaluating goodness-of-fit indexes for testing measurement invariance. Structural Equation Modeling-a Multidisciplinary Journal, 9(2), 233-255. https://doi.org/10.1207/s15328007sem0902_5

Coates, R., Ayers, S., \& de Visser, R. (2017). Factor structure of the Edinburgh Postnatal Depression Scale in a population-based sample. Psychological assessment, 29(8), 1016-1027. https://doi.org/10.1037/pas0000397

Costello, A. B., \& Osborne, J. (2005). Best practices in exploratory factor analysis: Four recommendations for getting the most from your analysis. Practical Assessment, Research \& Evaluation, 10, 1-9. https://doi.org/https://doi.org/10.7275/jyj14868

Cox, J. L., Holden, J. M., \& Sagovsky, R. (1987). Detection of postnatal depression: Development of the 10-item Edinburgh Postnatal Depression Scale. British Journal of Psychiatry, 150(6), 782-786. https://doi.org/10.1192/bjp.150.6.782

Cunningham, N. K., Brown, P. M., \& Page, A. C. (2015). Does the Edinburgh Postnatal Depression Scale measure the same constructs across time? [journal article]. Archives of Women's Mental Health, 18(6), 793-804. https://doi.org/10.1007/s00737-014-0485-9

de Bruin, G. P., Swartz, L., Tomlinson, M., Cooper, P. J., \& Molteno, C. (2004). The factor structure of the edinburgh postnatal depression scale in a South African peri-urban settlement. South African Journal of Psychology, 34(1), 113-121.

https://doi.org/https://doi.org/10.1177/008124 630403400107

Eastwood, J. G., Phung, H., \& Barnett, B. (2011). Postnatal depression and socio-demographic risk: Factors associated with Edinburgh Depression Scale scores in a metropolitan area of New South Wales, Australia. Australian and New Zealand Journal of Psychiatry, 45(12), 1040-1046.

https://doi.org/10.3109/00048674.2011.619160

Eberhard-Gran, M., Eskild, A., Tambs, K., Opjordsmoen, S., \& Samuelsen, S. O. (2001). Review of validation studies of the Edinburgh 
Postnatal Depression Scale. Acta psychiatrica Scandinavica, 104(4), 243-249.

https://doi.org/10.1034/j.1600-

0447.2001.00187.x

Garcia-Esteve, L., Ascaso, C., Ojuel, J., \& Navarro, P. (2003). Validation of the Edinburgh Postnatal Depression Scale (EPDS) in Spanish mothers. Journal of Affective Disorders, 75(1), 71-76. https://doi.org/10.1016/s0165-0327(02)00020-4

Guedeney, N., \& Fermanian, J. (1998). Validation study of the French version of the Edinburgh Postnatal Depression Scale (EPDS): New results about use and psychometric properties. European Psychiatry, 13(2), 83-89. https://doi.org/https://doi.org/10.1016/S09249338(98)80023-0

Gutiérrez-Zotes, A., Pujol, D. G., Arias, J. L., Santos, R. M., Esteve, L. G., Arbiol, E. G., Jover, M., Guillamat, R., Mayoral, F., \& Gornemann, I. (2018). Estructura factorial de la versión española de la Escala de Depresión Postnatal de Edimburgo. Actas Españolas de Psiquiatría, 46(5), 174-182.

Halbreich, U. (2005). The association between pregnancy processes, preterm delivery, low birth weight, and postpartum depressions--the need for interdisciplinary integration. American Journal of Obstetrics and Gynecology, 193(4), 1312-1322. https://doi.org/10.1016/j.ajog.2005.02.103

Hartley, C. M., Barroso, N., Rey, Y., Pettit, J. W., \& Bagner, D. M. (2014). Factor structure and psychometric properties of english and spanish versions of the edinburgh postnatal depression scale among Hispanic women in a primary care setting. Journal of Clinical Psychology, 70(12), 1240-1250. https://doi.org/10.1002/jclp.22101

Horn, J. L. (1965). A rationale and test for the number of factors in factor analysis. Psychometrika, 30, 179-185. https://doi.org/10.1007/bf02289447

Hu, L. T., \& Bentler, P. M. (1999). Cutoff criteria for fit indexes in covariance structure analysis: conventional criteria versus new alternatives. Structural Equation Modeling-a Multidisciplinary Journal, 6(1), 1-55. https://doi.org/10.1080/10705519909540118
Jardri, R., Pelta, J., Maron, M., Thomas, P., Delion, P., Codaccioni, X., \& Goudemand, M. (2006). Predictive validation study of the Edinburgh Postnatal Depression Scale in the first week after delivery and risk analysis for postnatal depression. Journal of Affective Disorders, 93(1-3), 169-176.

https://doi.org/10.1016/j.jad.2006.03.009

Jomeen, J., \& Martin, C. R. (2005). Confirmation of an occluded anxiety component within the Edinburgh Postnatal Depression Scale (EPDS) during early pregnancy. Journal of Reproductive and Infant Psychology, 23(2), 143-154.

https://doi.org/10.1080/02646830500129297

Jomeen, J., \& Martin, C. R. (2007). Replicability and stability of the multidimensional model of the Edinburgh Postnatal Depression Scale in late pregnancy. Journal of Psychiatric and Mental Health Nursing, 14(3), 319-324. https://doi.org/10.1111/j.1365-

2850.2007.01084.X

Kaiser, H., Hunka, S., \& Bianchini, J. (1971). Relating factors between studies based upon different individuals. Multivariate Behavioral Research, 6(4), 409-422.

https://doi.org/10.1207/s15327906mbr0604_3

King, P. A. L. (2012). Replicability of structural models of the Edinburgh Postnatal Depression Scale (EPDS) in a community sample of postpartum African American women with low socioeconomic status. Archives of Women's Mental Health, 15(2), 77-86. https://doi.org/10.1007/s00737-012-0260-8

Kubota, C., Inada, T., Nakamura, Y., Shiino, T., Ando, M., Aleksic, B., Yamauchi, A., Morikawa, M., Okada, T., Ohara, M., Sato, M., Murase, S., Goto, S., Kanai, A., \& Ozaki, N. (2018). Stable factor structure of the Edinburgh Postnatal Depression Scale during the whole peripartum period: Results from a Japanese prospective cohort study. Scientific Reports, 8(1), 17659.

https://doi.org/10.1038/s41598-018-36101-z

Kubota, C., Okada, T., Aleksic, B., Nakamura, Y., Kunimoto, S., Morikawa, M., Shiino, T., Tamaji, A., Ohoka, H., Banno, N., Morita, T., Murase, S., Goto, S., Kanai, A., Masuda, T., Ando, M., \& Ozaki, N. (2014). Factor structure of the Japanese Version of the 
Edinburgh Postnatal Depression Scale in the Postpartum Period. PLOS ONE, 9(8), e103941. https://doi.org/10.1371/journal.pone.0103941

Kwan, R., Bautista, D., Choo, R., Shirong, C., Chee, C., Saw, S. M., Chong, Y.-S., Kwek, K., Meaney, M. J., Rush, A. J., \& Chen, H. (2014). The Edinburgh Postnatal Depression Scale as a measure for antenatal dysphoria. Journal of Reproductive and Infant Psychology, 33(1), 28-41. https://doi.org/10.1080/02646838.2014.949642

Ledesma, R. D., Ferrando, P. J., \& Tosi, J. D. (2019). The use of exploratory factor analysis in RIDEP. Guidelines for Authors and Reviewers. Revista Iberoamericana de Diagnóstico - e Avaliação Psicológica, 3(52), 173-180. https://doi.org/10.21865/ridep52.3.13

Matthey, S., Fisher, J., \& Rowe, H. (2013). Using the Edinburgh postnatal depression scale to screen for anxiety disorders: Conceptual and methodological considerations. Journal of Affective Disorders, 146(2), 224-230. https://doi.org/10.1016/j.jad.2012.09.009

Mendoza, C., \& Saldivia, S. (2015). Actualización en depresión postparto: El desafío permanente de optimizar su detección y abordaje. Revista médica de Chile, 143(7), 887-894. https://scielo.conicyt.cl/pdf/rmc/v143n7/art10. pdf

Montazeri, A., Torkan, B., \& Omidvari, S. (2007). The Edinburgh Postnatal Depression Scale (EPDS): Translation and validation study of the Iranian version. BMC Psychiatry, 7(1), 11. https://doi.org/10.1186/1471-244X-7-11

Mook, J., Kleijn, W. C., \& Van der Ploeg, H. M. (1991). Symptom-positively and -negatively worded items in two popular self-report inventories of anxiety and depression. Psychological Reports, 69(2), 551-560. https://doi.org/10.2466/PR0.69.6.551-560

O'Hara, M. W. (1987). Post-partum 'blues,' depression, and psychosis: A review. Journal of Psychosomatic Obstetrics \& Gynecology, 7(3), 205-227. https://doi.org/10.3109/01674828709040280

Odalovic, M., Tadic, I., Lakic, D., Nordeng, H., Lupattelli, A., \& Tasic, L. (2015). Translation and factor analysis of structural models of
Edinburgh Postnatal Depression Scale in Serbian pregnant and postpartum women Web-based study. Women Birth, 28(3), e3135 .

https://doi.org/10.1016/j.wombi.2015.01.014

Oquendo, M., Lartigue, T., González-Pacheco, I., \& Méndez, S. (2008). Validez y seguridad de la Escala de Depresión Perinatal de Edinburgh como prueba de tamiz para detectar depresión perinatal. Perinatología y Reproducción Humana, 22(3), 195-202.

Petrozzi, A., \& Gagliardi, L. (2013). Anxious and depressive components of Edinburgh Postnatal Depression Scale in maternal postpartum psychological problems. Journal of Perinatal Medicine, 41(4), 343. https://doi.org/10.1515/jpm-2012-0258

Phillips, J., Charles, M., Sharpe, L., \& Matthey, S. (2009). Validation of the subscales of the Edinburgh Postnatal Depression Scale in a sample of women with unsettled infants. Journal of Affective Disorders, 118(1-3), 101112. https://doi.org/10.1016/j.jad.2009.02.004

Pitanupong, J., Liabsuetrakul, T., \& Vittayanont, A. (2007). Validation of the Thai Edinburgh Postnatal Depression Scale for screening postpartum depression. Psychiatry research, 149(1-3), 253-259.

https://doi.org/10.1016/j.psychres.2005.12.011

Pop, V. J., Komproe, I. H., \& van Son, M. J. (1992). Characteristics of the Edinburgh Post Natal Depression Scale in The Netherlands. Journal of Affective Disorders, 26(2), 105110. https://doi.org/10.1016/0165-0327(92)90041-4

Reichenheim, M. E., Moraes, C. L., Oliveira, A. S. D., \& Lobato, G. (2011). Revisiting the dimensional structure of the Edinburgh Postnatal Depression Scale (EPDS): Empirical evidence for a general factor. BMC Medical Research Methodology, 11(1), 93. https://doi.org/10.1186/1471-2288-11-93

Ross, L. E., Gilbert Evans, S. E., Sellers, E. M., \& Romach, M. K. (2003). Measurement issues in postpartum depression part 1: Anxiety as a feature of postpartum depression [journal article]. Archives of Women's Mental Health, 6(1), 51-57. https://doi.org/10.1007/s00737-002-0155-1 
Ruiz, M. A., \& San Martín, Á. (1993). Una implementación del procedimiento MAP para la determinación del número de factores. Psicothema, 5(1), 177-182.

Sharma, V., \& Mazmanian, D. (2014). The DSM5 peripartum specifier: Prospects and pitfalls. Archives of Women's Mental Health, 17(2), 171-173. https://doi.org/10.1007/s00737-013-0406-3.

Small, R., Lumley, J., Yelland, J., \& Brown, S. (2007). The performance of the Edinburgh Postnatal Depression Scale in English speaking and non-English speaking populations in Australia. Social Psychiatry and Psychiatric Epidemiology: The International Journal for Research in Social and Genetic Epidemiology and Mental Health Services, 42(1), 70-78. https://doi.org/10.1007/s00127-006-0134-3

Snaith, R. P., Constantopoulos, A. A., Jardine, M. Y., \& McGuffin, P. (1978). A Clinical Scale for the Self-assessment of Irritability. British Journal of Psychiatry, 132(2), 164-171. https://doi.org/10.1192/bjp.132.2.164

Swalm, D., Brooks, J., Doherty, D., Nathan, E., \& Jacques, A. (2010). Using the Edinburgh postnatal depression scale to screen for perinatal anxiety [journal article]. Archives of Women's Mental Health, 13(6), 515-522. https://doi.org/10.1007/s00737-010-0170-6

Töreki, A., Ando, B., Dudas, R. B., Dweik, D., Janka, Z., Kozinszky, Z., \& Kereszturi, A. (2014). Validation of the Edinburgh Postnatal Depression Scale as a screening tool for postpartum depression in a clinical sample in Hungary. Midwifery, 30(8), 911-918. https://doi.org/10.1016/j.midw.2014.02.008

Töreki, A., Andó, B., Keresztúri, A., Sikovanyecz, J., Dudas, R. B., Janka, Z., Kozinszky, Z., \& Pál, A. (2013). The Edinburgh Postnatal Depression Scale: Translation and antepartum validation for a Hungarian sample. Midwifery, 29(4), 308-315.

https://doi.org/https://doi.org/10.1016/j.midw. 2012.01.011

Tuohy, A., \& McVey, C. (2008). Subscales measuring symptoms of non-specific depression, anhedonia, and anxiety in the Edinburgh Postnatal Depression Scale. British Journal of Clinical Psychology, 47(2), 153-169. https://doi.org/10.1111/j.2044-

8260.2008.tb00463.x

Vázquez, M. B., \& Míguez, M. C. (2019). Validation of the Edinburgh postnatal depression scale as a screening tool for depression in Spanish pregnant women. Journal of Affective Disorders, 246, 515-521. https://doi.org/10.1016/j.jad.2018.12.075

Vega-Dienstmaier, J. M., Mazzotti Suárez, G., \& Campos Sánchez, M. (2002). Validación de una versión en español de la Escala de Depresión Postnatal de Edimburgo. Actas Españolas de Psiquiatría 30, 106-111.

Velicer, W. F., \& Jackson, D. N. (1990). Component analysis versus common factor analysis: Some Further Observations. Multivariate Behavioral Research, 25(1), 97114.

https://doi.org/10.1207/s15327906mbr2501_12

Vivilaki, V. G., Dafermos, V., Kogevinas, M., Bitsios, P., \& Lionis, C. (2009). The Edinburgh Postnatal Depression Scale: Translation and validation for a Greek sample. BMC Public Health, 9(1), 329. https://doi.org/10.1186/1471-2458-9-329

Zhong, Q., Gelaye, B., Rondon, M., Sanchez, S. E., Garcia, P. J., Sanchez, E., Barrios, Y. V., Simon, G. E., Henderson, D. C., Cripe, S. M., \& Williams, M. A. (2014). Comparative performance of Patient Health Questionnaire9 and Edinburgh Postnatal Depression Scale for screening antepartum depression. Journal of Affective Disorders, 162, 1-7. https://doi.org/10.1016/j.jad.2014.03.028

Zigmond, A. S., \& Snaith, R. P. (1983). The hospital anxiety and depression scale. Acta psychiatrica Scandinavica, 67(6), 361-370. https://doi.org/10.1111/j.16000447.1983.tb09716.x 